\title{
Analysis of Local Texture across Layers in Electron-Beam Melted (EBM) Ti-6Al- 4V via Electron Backscatter Diffraction (EBSD)
}

\author{
B. Saller ${ }^{1}$, J.R. Porter ${ }^{1}$, T. Van Den Vlekkert ${ }^{1}$, S. D. Walck ${ }^{2}$ \\ 1 University of California, Irvine, Dept. of Materials Science and Chemical Engineering, Irvine, \\ CA 92697 \\ 2 South Bay Technology, 1120 Via Callejon, San Clemente, CA 92673
}

Ti 6Al 4V (Ti 6-4) is an aerospace and biomedical titanium alloy of 6\% Aluminum, 4\% Vanadium, and $90 \%$ Titanium by weight, typically manufactured by casting, forging and heat treatment. During the electron beam melting (EBM) layer-build process, Ti-6Al-4V liquid droplets $(50 \mu \mathrm{m}$ in size on average) are deposited onto the growing part as a body-centered $\beta$-cubic (BCC) alloy. On cooling, a transformation to stable hexagonal laths occurs within the prior $\beta$-cubic grains. ${ }^{1}$ Our investigation is to establish whether these prior $\beta$-grains form with either a random or preferred crystallographic orientation, as the material grows layer-by-layer.

A plane of the material showing the layers was analyzed in order to understand the process of layer building in a direction normal to the layer planes (Fig. 1). This study used EBSD to characterize the orientation of the grain structure by observation of the diffraction patterns created by scattered electrons. $^{2}$ The objective of this study is continued improvement of the EBM build process as well as selection of ideal build parameters, resulting in a cost effective solution to low volume production of parts.

The specimen preparation challenge was to prepare samples that revealed the microstructure together with a high indexing rate during EBSD mapping. Samples were mechanically polished using SiC paper and diamond lapping films ending with $0.25 \mu \mathrm{m}$ diamond suspension followed by colloidal silica. The final step was ion polishing in order to remove the damaged layer created by mechanical polishing as well as to etch the sample. A South Bay Technology IBS/e equipped with a KRI KDC10 Kaufman ion source was used for ion polishing in two steps. The parameters were as follows: 1) $15 \mathrm{~min}, 600 \mathrm{~V}, 5 \mathrm{~mA}, 80^{\circ}$ (relative to the normal to the sample surface), 2) $30 \mathrm{~min}, 250 \mathrm{~V}, 5 \mathrm{~mA}$, $85^{\circ}$.

EBSD maps were obtained using an Oxford Nordlys camera interfaced to a Zeiss Ultra Plus SEM. ${ }^{3}$

The EBSD map in Figure 2 shows the orientations of each individual grain by assigning colors. The colors represent Miller Indices of planes parallel to the sample surface, which is the IPF-Z pattern. The complete pole figure in Figure 3 shows six distinct clusters corresponding to the (0001)) planes of hexagonal Ti. Sectioning each region of the EBSD map and obtaining that region's pole figure is shown in Figure 4. These are indeed the layers due to layer thickness in the EBM build process being on the order of 100 microns that matches the thickness shown on the EBSD map.

Isolating each layer (region) and obtaining their pole figures resulted in six clusters being shown in each pole figure. Since each layer represents approximately one prior $\beta$-cubic grain, we can say that there is a preferred orientation (or texture). Given the $(0001) / /(110)$ orientation relationship for the beta to alpha transformation, the (0001) poles can be associated with the (110) poles of the prior beta shows that all the prior cubic grains are close to a standard cube orientation. The inference of this 
data is that there is a preferred orientation (texture) that spans multiple layers of the layer-build process and that, in this particular region; the prior beta structure had a cube texture.

[1] Adnan Safdar, Liu-Ying Wei, "Microstructures of Electron Beam Melted (EBM) Biomaterial Ti6Al-4V," Mater. Res. Soc. Symp. Proc., 1132. 2009.

[2] Dave Prior, Gareth Seward, Michel Bestmann, Sandra Piazolo, "EBSD at High Temperatures in Metals and Minerals," Microscopy Microanalysis, 9. 2003.

[3] The authors acknowledge the use of the Carl Zeiss Center of Excellence at UCI and the provision of equipment by Oxford Instruments and South Bay Technology.

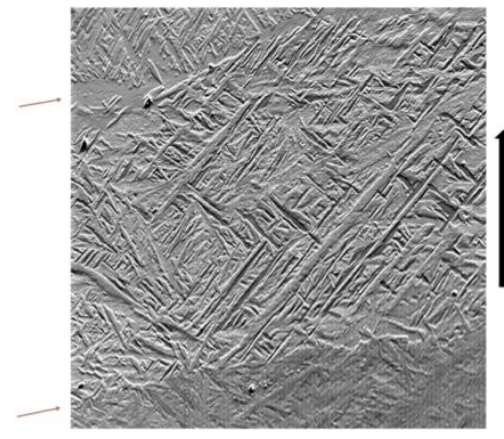

Fig. 1 Layer-built Ti 6 Al 4 V

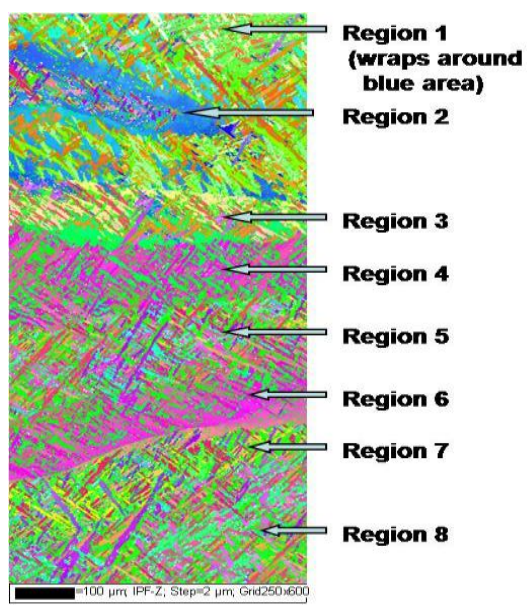

Fig. 2. IPF-Z map of sample

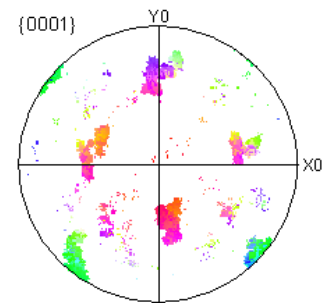

Fig. 3 Pole Figure from map
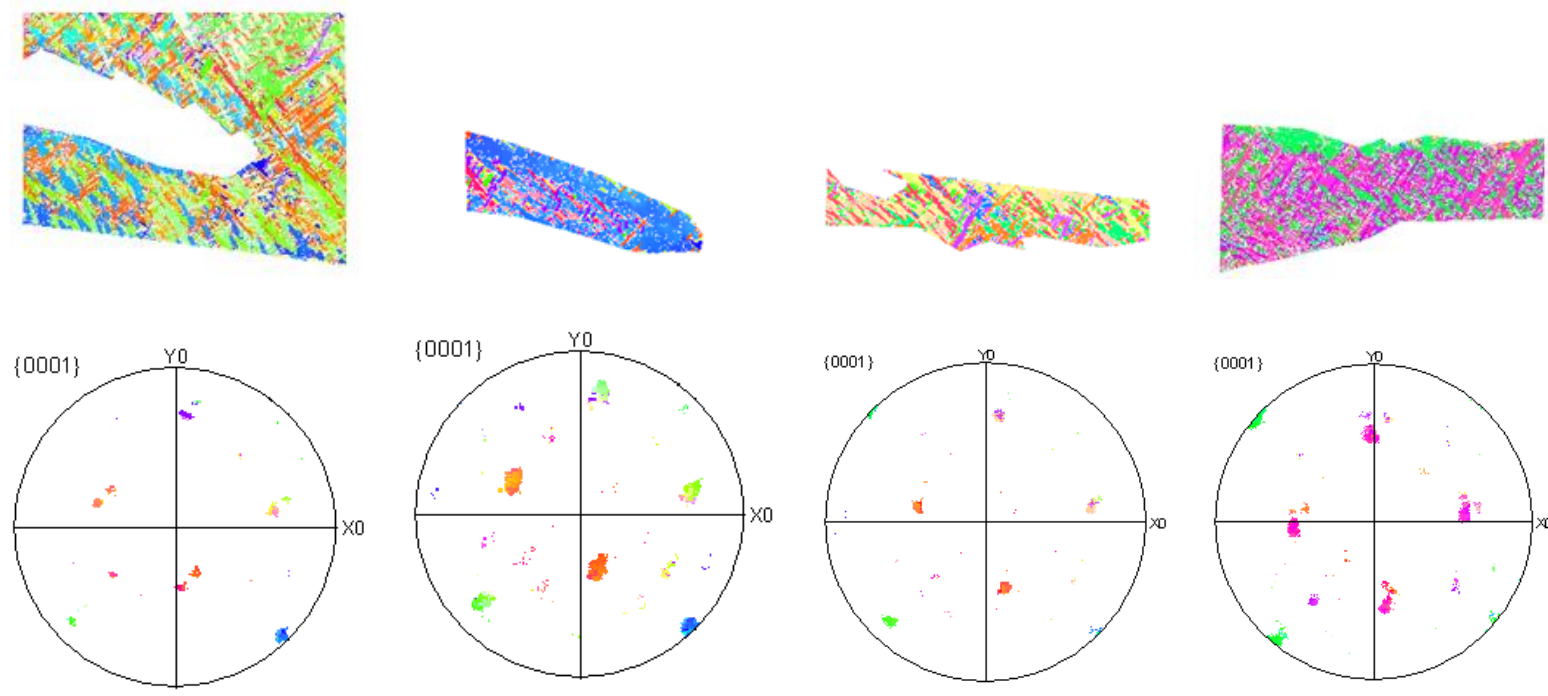

Region 3

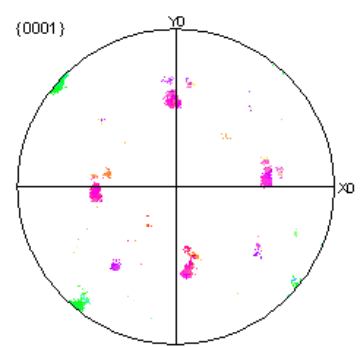

Region 4

Fig 4. Pole figures from four regions of EBSD map 